\title{
Continuous-Wavelet Transform for Fault Location in Distribution Power Networks: Definition of Mother Wavelets Inferred From Fault Originated Transients
}

\author{
Alberto Borghetti, Senior Member, IEEE, Mauro Bosetti, Mauro Di Silvestro, Carlo Alberto Nucci, Fellow, IEEE, \\ and Mario Paolone, Member, IEEE
}

\begin{abstract}
The paper presents a fault location procedure for distribution networks based on the wavelet analysis of the fault-generated traveling waves. In particular, the proposed procedure implements the continuous wavelet analysis applied to the voltage waveforms recorded during the fault in correspondence of a network bus. In order to improve the wavelet analysis, an algorithm is proposed to build specific mother wavelets inferred from the fault-originated transient waveforms.

The performance of the proposed algorithm are analyzed for the case of the IEEE 34-bus test distribution network and compared with those achieved by using the more traditional Morlet mother wavelet.
\end{abstract}

Index Terms-Continuous wavelet transform, distribution network, EMTP simulations, fault location, mother wavelet, power quality.

\section{INTRODUCTION}

$\mathbf{P}$ OWER quality of distribution networks, with particular reference to the number and duration of short and long interruptions, depends on the annual number of faults and on the relevant restoration times. The availability of accurate fault location techniques can result in important reduction of restoration times [1].

The fault location problem has been extensively investigated and several approaches have been proposed in the literature. These approaches can be grouped into the following main categories: 1) methods based on impedance measurement (e.g., [2]-[4]); 2) methods based on the analysis of fault-originated traveling waves (e.g., [5]-[9]). It is worth mentioning that expert systems, often based on the use of neural networks, have been also proposed (e.g., [10]-[12]).

Impedance measurement methods rely essentially on the analysis at power frequency of voltage and current fault signals. Fault location in transmission lines is the typical application field of these techniques in view of the significant line length and simple network topology. Their application to distribution

Manuscript received May 22, 2007; revised September 29, 2007. This work was a part of a research program supported by CESI, Italy. Paper no. TPWRS00356-2007.

The authors are with the Department of Electrical Engineering, University of Bologna, Bologna, Italy (e-mail: alberto.borghetti@mail.ing.unibo.it; mauro.bosetti@mail.ing.unibo.it; mauro.disilvestro@mail.ing.unibo.it; carloalberto.nucci@mail.ing.unibo.it; mario.paolone@mail.ing.unibo.it).

Digital Object Identifier 10.1109/TPWRS.2008.919249 networks, characterized by multibranched radial topologies, may result in a decrease of the location accuracy.

Traveling wave methods involve the measurements of highfrequency components as well as the implementation of complex signal analysis techniques. They rely on the analysis of the high-frequency components of fault-oriented transients which are rather uninfluenced by the fault type and impedance [7]. Their use in distribution network has been already proposed typically coupled with the use of the discrete wavelet analysis (e.g., [6], [8]).

The method presented in this paper belongs to category ii) and is based on the extension of the algorithm presented in [13]. Voltage transients generated by the fault are analyzed by using the continuous wavelet transformation (CWT); the proposed CWT analysis, performed in frequency domain, is aimed at determining peculiar frequencies that can be used to infer the fault location assuming the network topology and line conductor geometry known.

In order to overcome some limitation and to improve the performances of the method proposed in [13], this paper proposes an algorithm to build specific mother wavelets directly inferred from the recorded fault-originated voltage transients. The influence of such a fault-inferred mother wavelet on the fault location accuracy is analyzed, and the results compared with those obtained by using the traditional Morlet mother wavelet, which is one of the several mother wavelets proposed in the literature (e.g., [14]-[19]).

The paper is structured as follows. Section II describes the information that can be inferred from fault-originated traveling waves and the adequacy of different signal processing techniques that can be adopted to analyze fault transients. Section III illustrates the algorithm for the definition of transient-based mother wavelets and Section IV shows some application referring to the IEEE 34-bus distribution system [20].

\section{Proposed Traveling-WaVe ApProACH}

The proposed approach is based on the identification of some characteristic frequencies associated with specific paths in which the fault-originated traveling waves are propagating. These characteristic frequencies can be identified by means of adequate signal analysis techniques applied to the voltage or current waveforms recorded at an observation point (typically located at the lower voltage terminals of the transformer feeding the distribution network). 


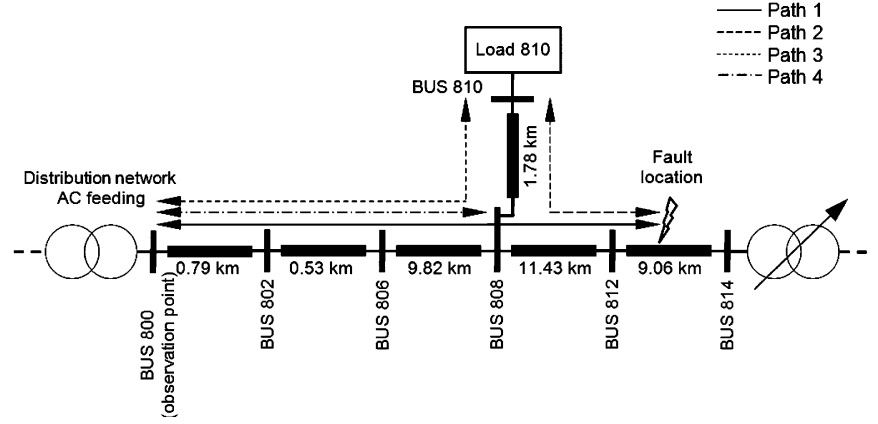

Fig. 1. Example of the paths covered by the traveling waves associated to a fault location placed between buses 812 and 814 of the first section of the main feeder of the IEEE 34-bus distribution system.

\section{A. Fault-Originated Traveling-Wave Paths and Associated Characteristic Frequencies}

Fault-originated traveling waves propagate along the network and reflect at line terminations, junctions between feeders, and the fault location. The relevant reflection coefficients depend to the line surge impedances, the impedances of power components connected to the network terminations and to the fault impedance value.

A certain number of paths $p$, covered by the traveling waves, can be associated to the observation point $m$ where the voltage or current waveforms are measured.

Assuming a network topology characterized by a main feeder and some laterals (radial configuration), the number of paths is equal to the number of network laterals plus the number of feeder-lateral junctions. As an example, Fig. 1 shows a fault location placed between buses 812 and 814 of the first section of the main feeder of the IEEE 34-bus distribution system (additional details relevant to this model are reported in Section IV). Four different paths can be identified and paths \#1, \#3 and \#4 can be associated to the relevant traveling waves and are contained in the voltage or current waveforms recorded at the observation point placed in correspondence of the bus 800 (medium voltage side of the feeding substation).

Each path $p$ can be associated to a number of characteristic frequencies, one for each of the traveling-wave propagation modes [21], [22]. Indeed, it is worth noting that the propagation of traveling waves in multi-conductor lines involves the presence of different propagation speeds. Therefore, the identification of characteristic frequencies related to each path $p$ is separately carried out for the various propagation modes. In order to apply the modal transformation matrixes in time-domain, the modal transformation matrix should be real and their calculation in this paper is performed using the line constant routine of the EMTP [22].

Assuming the network topology and the traveling wave speeds of the various propagation modes are known, frequency $f_{p, i}$ of mode $i$ through path $p$ can be evaluated a priori as

$$
f_{p, i}=\frac{v_{i}}{n_{p} L_{p}}
$$

where $v_{i}$ is the traveling speed of the $i$ th propagation mode, $L_{p}$ is the length of the $p$ th path and $n_{p}(\in \mathbf{N})$ is the number of times needed for a given traveling wave to propagates along path $p$ before attain again the same polarity. $p-1$ values are used to identify the faulted section and the remaining one to identify the fault distance between observation point $m$ and the fault location.

In order to associate a characteristic frequency to each path, we can see the fault as a step-function source triggered by the fault occurrence. The fault-generated step wave travels along the network and is reflected in correspondence to the abovementioned path extremities. Each extremity is characterized by specific voltage reflection coefficient, namely:

- extremities where a power transformer is connected can be considered as open circuits and the relevant reflection coefficient is close to +1 ;

- extremities that correspond to a junction between more than two lines are characterized by a negative reflection coefficient;

- the reflection coefficient of the extremity where the fault is occurring is close to -1 , as the fault impedance value is lower than the line surge impedance.

The coefficient $n_{p}$ depends on the sign of the reflection coefficients of the two path extremities, namely $n_{p}$ is equal to 2 or to 4 if the reflection coefficients have the same or opposite sign, respectively.

\section{B. Signal Analysis Techniques for the Identification of Characteristic Frequencies of Traveling-Waves Paths}

Current or voltage fault transient signals are composed by superimposing the industrial frequency waveform (constant low frequency component of large duration) and the transient disturbance caused by the fault (time-varying high frequency component of short duration). The resulting signal is therefore characterized by a continuous spectrum due to its time-variant properties. The identification of characteristic frequencies $f_{p, i}$ by means of traditional operators, such as the fast Fourier transform (FFT), is certainly not appropriate. Indeed, such an operator analyzes the signal with a constant frequency resolution that depends to the width of the chosen observation time window. ${ }^{1}$

In view of the above, the identification of characteristic frequencies $f_{p, i}$ should be accomplished by using appropriate signal analysis techniques that allow the adjustment of the signal spectrum versus time. Such a requirement is fulfilled by implementing the so-called time-frequency representations (TFRs) [23]. In particular, a signal TFR links a one-dimensional time signal $x(t)$ to a bi-dimensional function of time and frequency, $T_{x}(t, f)$. Typical examples of linear TFRs are the short time Fourier transform (STFT) and the wavelet transform.

As known, STFT is a windowed Fourier transform in which the observation interval is divided into a given number of subintervals. For each subinterval, STFT is computed according to the following equation:

$$
T_{\mathrm{STFT}}(t, f)=\int_{-\infty}^{+\infty} x(\tau) w(t-\tau) e^{-j \omega \tau} d \tau
$$

${ }^{1}$ In order to provide an example, let consider that frequencies $f_{p, i}$ usually ranges from few $\mathrm{kHz}$ to few tens of $\mathrm{kHz}$ and are contained in a time window of the fault signals of a typical duration of $1 \mathrm{~ms}$. Therefore, the application of the traditional FFT to the fault signal results in a frequency resolution of $1 \mathrm{kHz}$ that is certainly inadequate to correctly identify frequencies $f_{p, i}$. 
where $w(\tau)$ is the windowing function that defines the length of the subinterval. Similarly to the Fourier transform, the main characteristic of the STFT is that the time-frequency resolution is constant and equal to the duration of each subinterval. Therefore, it is not the more appropriate tool for the analysis of fault signals.

The wavelet transform, on the other hand, is a TFR that allows a good frequency resolution at low frequencies and a good time resolution at high frequencies [24]. In particular, it allows for the analysis of high frequency components very close to each other in time and low frequency components very close each other in frequency. These properties are indeed suitable for the study of transient waveforms produced by faults.

The proposed fault location approach is based on the use of a CWT. The CWT of signal $x(t)$ is, as known, the integral of the product between $x(t)$ and the so-called daughter-wavelets, which are time translated and scale expanded/compressed versions of a finite energy function $\psi(t)$, called mother wavelet. This transformation, equivalent to a scalar product, produces wavelet coefficients $C(a, b)$ that represent the TFR bi-dimensional function of time and frequency $T_{x}(t, f)$. Coefficients $C(a, b)$ can be seen as similarity indexes between the signal and the daughter wavelet located at position $b$ (time shifting factor) with scale $a$.

The analyzed part $s(t)$ of the recorded signal $x(t)$, that corresponds to a voltage or current fault-transient, is usually characterized by a short duration of few milliseconds. Such a duration corresponds to the product between sampling time $T_{s}{ }^{2}$ and number of recorded samples $N$. Therefore, in the numerical implementation of the CWT applied to signal $s(t)$, the elements of matrix $C(a, b)$ of are given by

$$
\begin{aligned}
C(a, b) & =C\left(a, i T_{s}\right) \\
& =T_{s} \frac{1}{\sqrt{|a|}} \sum_{n=0}^{N-1} \psi^{*}\left[\frac{(n-i) T_{s}}{a}\right] s\left(n T_{s}\right)
\end{aligned}
$$

where parameter $a$ corresponds to the scale factor and product $i \cdot T_{s}$ corresponds to the so-called time shifting factor $b$. It is worth nothing that if the center frequency of the mother wavelet $\psi(t)$ is $F_{0}$, the one of the daughter-wavelet $\psi(a t)$ is $F_{0} / a$.

The sum of the squared values of all coefficients belonging to the same scale, which are denoted as CWT signal energy $E_{\text {cwt }}(a)$, identifies a "scalogram" which provides the weight of each frequency component [25]

$$
E_{\text {cwt }}(a)=\sum_{n=0}^{N-1}\left(C\left(a, n T_{s}\right)\right)^{2} .
$$

The identification of the characteristic frequencies $f_{p, i}$ associated to the fault location is realized by inspecting the relative maximum peaks of the obtained scalogram $E_{\mathrm{cwt}}(a)$.

\section{First Application Example: Balanced Solid Fault}

As a first example, the proposed fault location procedure is applied to the case of a three-phase balanced solid fault at bus 812 of the IEEE 34-bus distribution system simulated by means of a model implemented in the EMTP-RV environment [26],

\footnotetext{
${ }^{2}$ All the examples provided in the paper make reference to a sampling frequency equal to $10 \mathrm{MHz}$.
}

TABLE I

THEORETICAL AND CWT-IDENTIFIED CHARACTERISTIC FREQUENCIES Relevant to the Propagation Paths For a Three-Phase Solid Fault LoCATED AT Bus 812 OF Fig. 1. THE RESUlTS MaKe REFERENCE tO THE Morlet Mother WaVElet. The ANALYSIS ReFERS to Propagation Mode 1

\begin{tabular}{|c|c|c|c|}
\hline Path & $\begin{array}{c}\text { Path } \\
\text { length } \\
n_{p} \cdot L_{p} \\
(\mathrm{~km})\end{array}$ & $\begin{array}{c}\text { Theoretical frequencies } f_{p, i} \\
\text { (traveling wave refer to propagation } \\
\text { mode } 1) \\
(\mathrm{kHz})\end{array}$ & $\begin{array}{c}\text { CWT identified } \\
\text { frequency } \\
(\mathrm{kHz})\end{array}$ \\
\hline $800-812$ & $4 \times 22.57$ & 3.29 & 3.70 \\
\hline $800-808$ & $4 \times 11.14$ & 6.68 & - \\
\hline $800-810$ & $2 \times 12.92$ & 11.52 & 9.60 \\
\hline
\end{tabular}

[27]. Additional details on such a system, relevant transmission line propagation modes and implementation are given in Section IV.

Locating the observation point $m$ at bus 800 and the fault at bus 812 , three traveling-waves are contained in the fault transient. They are characterized by a common path-terminal, bus 800 , and other three path-terminals corresponding to buses 812 (path \#1), 808 (path \#2) and 810 (path \#3).

As reported above, if we consider, in a first approximation, the fault as a step-function source triggered by the fault occurrence, the resulting voltage transient observed at bus 800 is the sum of three square waves each one characterized by a main frequency given by (1). Table I shows the theoretical frequency values obtained by applying (1) to the considered example.

Table I also shows the characteristic frequency values obtained by applying the CWT-analysis using with the Morlet mother wavelet to the voltage transient recorded at the medium voltage side of the feeding substation (bus 800).

The CWT-analysis is performed in a time window of $2 \mathrm{~ms}$ and refers to propagation mode 1 . The specific characteristics of line propagation modes are given in Table III of Section IV.

Fig. 2 shows the obtained scalogram relevant to the signal energy values $E_{\text {cwt }}(a)$. As it can be seen from Fig. 2, the CWT analysis is able to identify the frequencies related to path \#1 and path \#3, whilst it is unable to identify path \#2. The frequency associated with the second path is hidden by the other frequencies due to the large filter amplitude related to the adopted mother wavelet.

The location error is defined as

$$
e_{\%}=\frac{100}{L_{p *}}\left|L_{p *}-\frac{\nu_{i}}{n_{p *} \cdot f_{p *, i}^{\mathrm{CWT}}}\right|
$$

where $L_{p *}$ is the length of path $p *$ between the observation bus and the fault location, $v_{i}$ is the propagation speed of mode $i, n_{p *}$ is equal to 4 and $f_{p *, i}^{\mathrm{CWT}}$ is the CWT-identified frequency relevant to path $p *$.

The location error for the considered fault achieved by the CWT analysis is equal to $10.9 \%$.

\section{DeFinition OF Mother WAVELETS INFERRED FROM FAULT-ORIGINATED TRANSIENTS}

\section{A. Requirements for the Definition of a Mother Wavelet}

As known, the CWT allows for the adoption of arbitrary mother wavelets that have to comply with the so-called admis- 


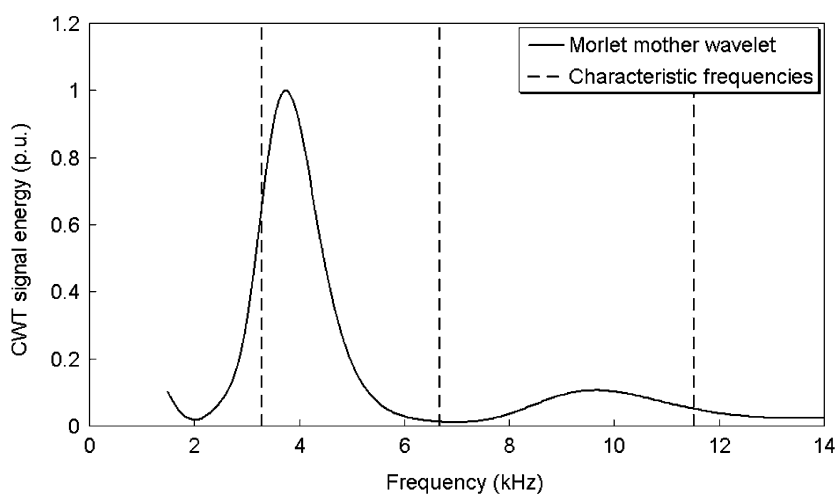

Fig. 2. Results of the CWT analysis of the mode 1 of the voltage transient recorded at bus 800 for a three-phase solid fault at bus 812 . The values are in per-unit with respect to the maximum.

sibility condition

$$
C_{\psi}=\int_{-\infty}^{+\infty} \frac{|\Psi(\omega)|^{2}}{\omega} d \omega<\infty .
$$

Sufficient conditions (SC) to satisfy (6) are SC-a) mean value of $\psi(t)$ equal to zero, namely $\int_{-\infty}^{+\infty} \psi(t) d t=0$; SC-b) fast decrease to zero of $\psi(t)$ for $t \rightarrow \pm \infty$.

If the CWT backward transformation (7), i.e., the signal reconstruction, must be guaranteed, the choice of the number and spacing of scales $a$ as well as the choice of the mother wavelet, should comply with the so-called orthogonality condition (e.g., [28])

$$
f(t)=\frac{1}{C_{\psi}} \int_{-\infty}^{+\infty} \int_{-\infty}^{+\infty} C(a, b) \psi_{a, b}(t) \frac{d a d b}{a^{2}} .
$$

The CWT adopted in the proposed fault location procedure does not require to satisfy the orthogonality condition which is needed if time-domain fault location approaches (e.g., [7], [8]), based on reconstruction of the fault transient signal related to each characteristic frequency, are used.

\section{B. Algorithm for the Construction of a Mother Wavelet Inferred From Fault-Originated Transients}

We start from the concept that the CWT can be considered as a filtering process based on the scalar product between daughter wavelets and the analyzed signal. The maximization of such a scalar product is related to the similarity between the mother wavelet and the signal itself. Therefore, the proposed approach builds the mother wavelet by applying the above sufficient conditions to the initial part of the fault transient waveform.

The algorithm developed for building the mother wavelet is composed by the following steps, which will be described next. In order to illustrate the implementation of each step, the description refers to the case of a three-phase solid fault located in correspondence of bus 814 of the IEEE 34-bus distribution system.

1) Being $s(t)$ the fault transient waveform relevant to a specific propagation mode, $\bar{s}(t)$ is extracted as the initial part

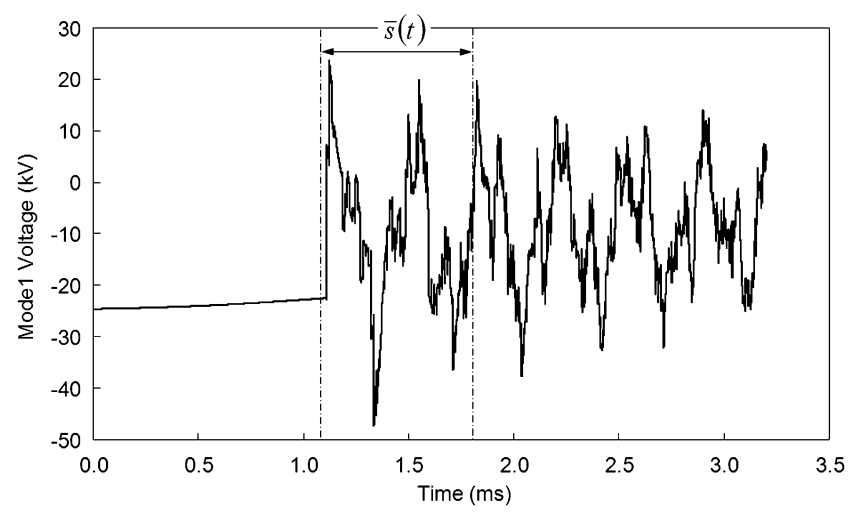

Fig. 3. Fault voltage transient waveform of the propagation mode 1 observed at bus 800 of the IEEE 34-bus distribution system for a three-phase solid fault in 814 ; the selection of $\bar{s}(t)$ used to build the mother wavelet.

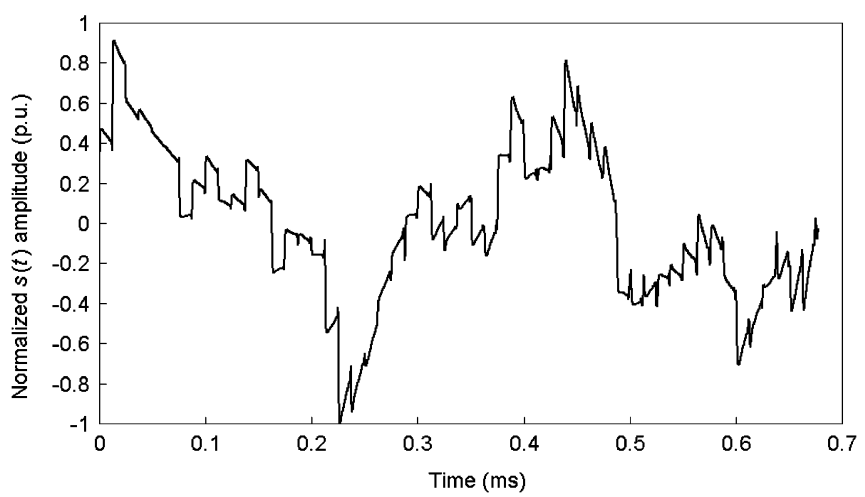

Fig. 4. Normalization and shift of $\bar{s}(t)$.

of $s(t)$. Function $\bar{s}(t)$ is used to build the mother wavelet $\psi(t)$ and starts from the fault-occurrence time with a duration $\Delta t$ that corresponds to the minimum expected frequency content of the analyzed signal. Fig. 3 shows the voltage waveform of the propagation mode 1 observed at bus 800 and the selection of the part of the fault transient waveform $\bar{s}(t)$ used to build the mother wavelet.

2) $\bar{s}(t)$ is then normalized with respect to its maximum value. To satisfy the above mentioned SC-a, $\bar{s}(t)$ is then shifted in order to obtain a mean value equal to zero. Fig. 4 shows the modified signal $\bar{s}(t)$ according with this step of the algorithm.

3) Finally, in order to satisfy SC-b, the mother wavelet $\psi(t)$ is built as a series of several $k \Delta t$-shifted $\bar{s}(t)$ multiplied by an exponential decay characterized by a time constant $\tau$

$$
\psi(t)=\left(\sum_{k \in N} \bar{s}(t+k \Delta t)+\bar{s}(t-k \Delta t)\right) e^{-\tau t^{2}}
$$

with

$$
\bar{s}(t)= \begin{cases}\bar{s}(t) & 0 \leq t \leq \Delta t \\ 0 & t \leq 0 ; t \geq \Delta t .\end{cases}
$$

Fig. 5 shows the obtained mother wavelet.

The obtained mother wavelet $\psi(t)$ given by (8) is indeed a mother wavelet because its construction is in accordance with 


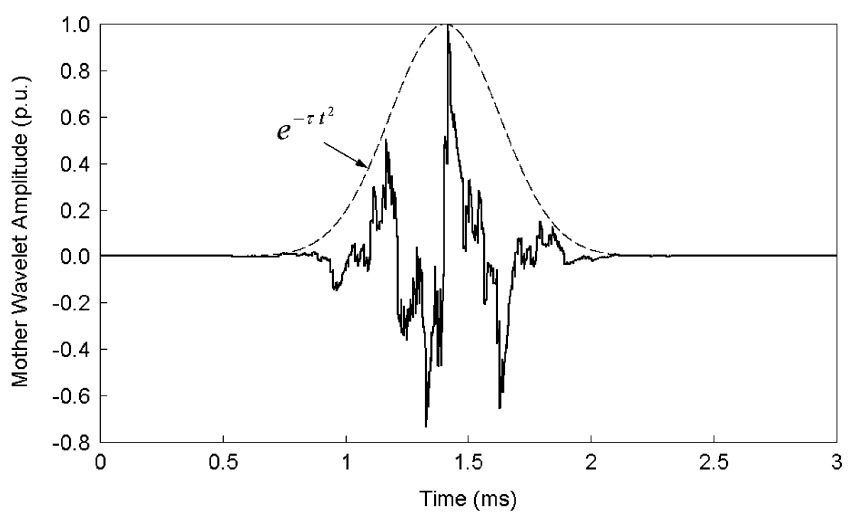

Fig. 5. Transient-based mother wavelet built from $\bar{s}(t)$ of Fig. $3\left(\tau=10^{-7}\right)$.

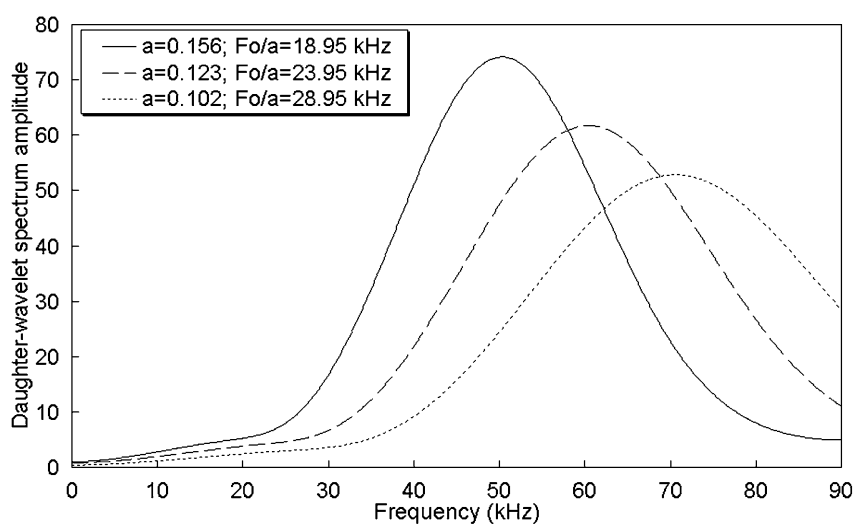

Fig. 6. Frequency domain spectrums of three daughter-wavelet (referring to three different scales) of the transient-based mother wavelet of Fig. 5.

the two sufficient condition (SC-a and SC-b) that satisfy the admissibility condition.

As known, the wavelet analysis consists in a filtering process of the analyzed signal by means of subsequent constant-energy shifting-filter given by the daughter wavelets

$$
F(C(a, b))=\sqrt{a} \Psi^{*}(a \cdot \omega) X(\omega)
$$

where $F(C(a, b)), X(\omega)$ and $\Psi(\omega)$ are the Fourier transforms of $C(a, b)$, the fault signal $x(t)$ and the daughter wavelet $\psi(t)$ respectively. According to this wavelet analysis basic concept, Fig. 6 shows the frequency-domain spectrum amplitudes of three daughter wavelets (referring to three different scales) of the transient-based mother wavelet of Fig. 5. As it can be seen, the three curves of Fig. 6 clearly refer to a constant-energy shifting-filter.

Fig. 7 shows the comparison of the results obtained with the CWT analysis by using the fault-inferred and Morlet mother wavelets. The identified frequencies, as well as the relevant paths, are reported in Table II.

As for the case illustrated in the previous section, the CWT analysis referring to the Morlet mother wavelet is able to detect only the frequencies associated with the first and second paths while the frequency peak associated with the third path appears hidden by the second peak. On the contrary, the CWT referring to the transient-inferred mother wavelet allows the identification of all the three frequencies.

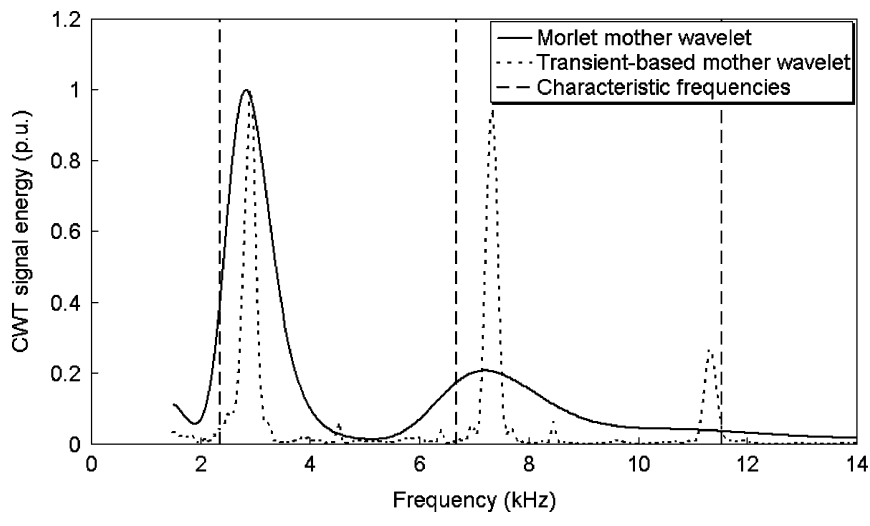

Fig. 7. Comparison between the results of the CWT analysis performed with Morlet and fault-inferred mother wavelets of mode 1 of the voltage transient recorded at bus 800 for a three-phase solid fault at bus 814 . The values are in per-unit with respect to the maximum.

TABLE II

CHARACTERISTIC AND CWT IDENTIFIED-FREQUENCIES RELEVANT TO THE PROPAGATION PATHS FOR A THREE-PHASE SOLID FAULT LOCATED AT BUS 814 of Fig. 1. THE ANALYSIS REFERS tO the PROPAGATION MOdE 1

\begin{tabular}{|c|c|c|c|c|}
\hline \multirow[b]{2}{*}{ Path } & \multirow{2}{*}{$\begin{array}{l}\text { Path } \\
\text { length } \\
n_{p} \cdot L_{p} \\
(\mathrm{~km})\end{array}$} & \multirow{2}{*}{$\begin{array}{l}\text { Theoretical } \\
\text { frequencies } f_{p, i} \\
\text { (traveling wave refer } \\
\text { to propagation } \\
\text { mode 1) } \\
(\mathrm{kHz})\end{array}$} & \multicolumn{2}{|c|}{$\begin{array}{l}\text { CWT identified frequency } \\
\qquad(\mathrm{kHz})\end{array}$} \\
\hline & & & $\begin{array}{c}\text { Transient- } \\
\text { based mother } \\
\text { wavelet }\end{array}$ & $\begin{array}{l}\text { Morlet } \\
\text { mother } \\
\text { wavelet }\end{array}$ \\
\hline $800-814$ & $4 \times 31.63$ & 2.35 & 2.90 & 2.80 \\
\hline $800-808$ & $4 \times 11.14$ & 6.68 & 7.35 & 7.20 \\
\hline $800-810$ & $2 \times 12.92$ & 11.52 & 11.35 & - \\
\hline
\end{tabular}

TABLE III

Value of the Modal Parameters Calculated at $1 \mathrm{KHz}$ oF THE CONSIDERED OVERHEAD LINE CONFIGURATION, GROUND RESISTIVITY EQUAL TO $100 \Omega \mathrm{m}$

\begin{tabular}{|c|c|c|c|c|c|}
\hline mode & $\begin{array}{c}r \\
(\Omega / \mathrm{km})\end{array}$ & $\begin{array}{c}l \\
(\mathrm{mH} / \mathrm{km})\end{array}$ & $\begin{array}{c}c \\
(\mu \mathrm{F} / \mathrm{km})\end{array}$ & $\begin{array}{c}z_{c} \\
(\Omega)\end{array}$ & $\begin{array}{c}\text { Propagation } \\
\text { speed } \\
(\mathrm{km} / \mathrm{s})\end{array}$ \\
\hline 0 & 0.984 & 2.367 & $5.823 \cdot 10^{-03}$ & 637.99 & $2.693 \cdot 10^{+05}$ \\
\hline 1 & 0.136 & 0.908 & $1.243 \cdot 10^{-02}$ & 270.27 & $2.976 \cdot 10^{+05}$ \\
\hline 2 & 0.065 & 0.875 & $1.273 \cdot 10^{-02}$ & 262.17 & $2.997 \cdot 10^{+05}$ \\
\hline
\end{tabular}

\section{APPlication EXAMPles of THE PROPOSED Algorithm FOR FAULT LOCATION IN DISTRIBUTION NETWORKS}

The previous examples refer to the case of balanced solid faults. In what follows, the performances of the proposed algorithm are tested against for the cases of: 1) unbalanced faults, 2) variable values of fault impedance; 3 ) different exponential decay time of the mother wavelet; and 4) different fault locations along the IEEE 34-bus distribution system, as described in what follows.

\section{A. Adapted IEEE 34-Bus Distribution Systems Adapted for This Study}

The IEEE 34-bus test feeder is composed by branches characterized by different conductor configurations. In order to simplify the network configuration, the following assumptions have been made: 1) all the branches of the network are composed by overhead lines which conductor configuration is the "ID \#500" reported in [20, Fig. 1] (three-phase plus neutral); 2) the network 


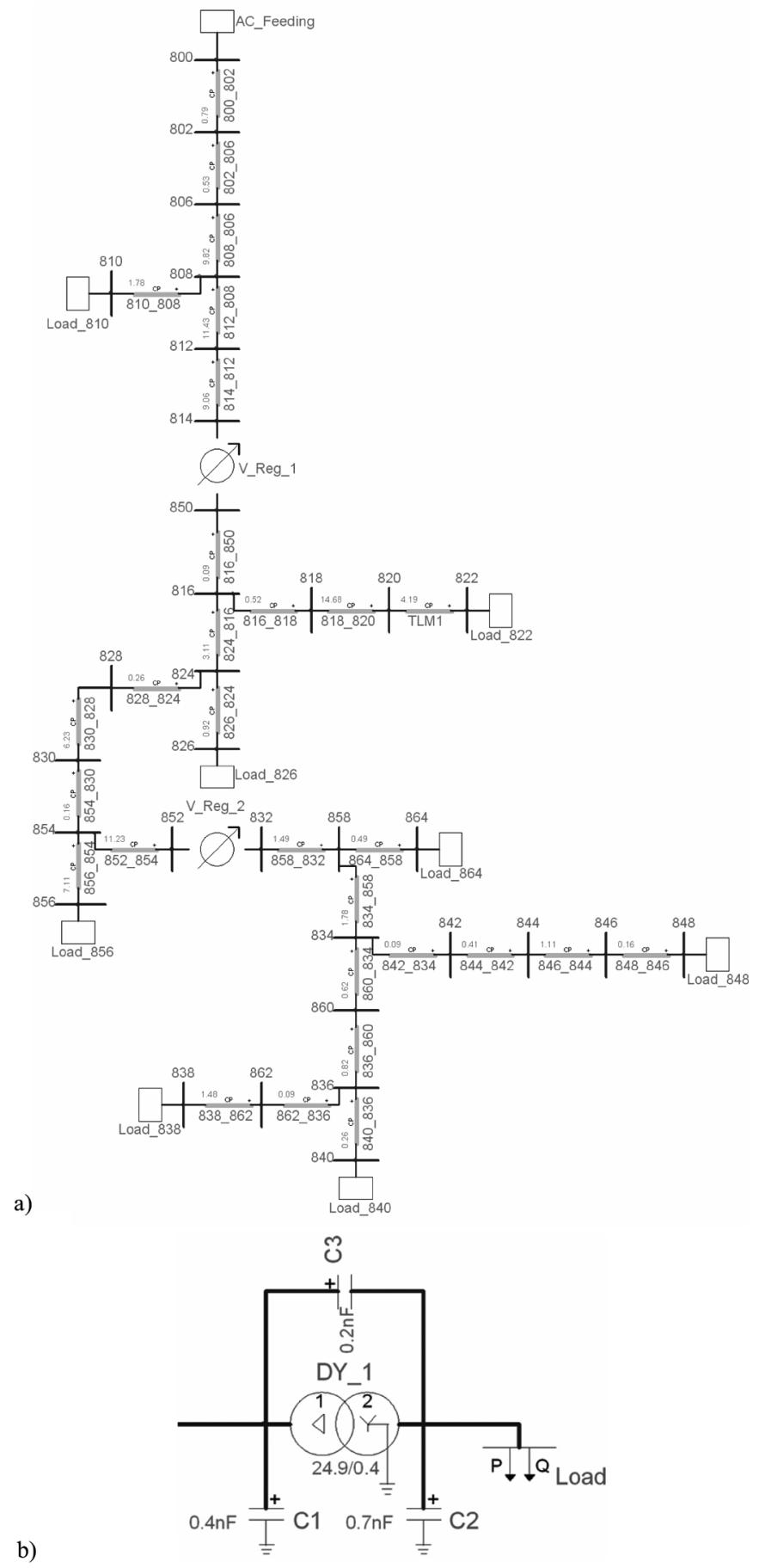

Fig. 8. Analyzed case study. (a) IEEE 34-bus distribution system implemented in EMTP-RV. (b) Transformer model.

loads are assumed located in correspondence of the line terminations and connected by means of distribution power transformers. Fig. 8(a) shows the IEEE 34-bus distribution system topology implemented in the EMTP-RV simulation environment.

Concerning the power distribution transformers, they are represented by means of the parallel of a $50 \mathrm{~Hz}$ standard model and a $\Pi$ of capacitances in order to represent, in a first approximation, its response to transients at a frequency range around 100
TABLE IV

CHARACTERISTIC AND CWT IDENTIFIED FREQUENCIES RELEVANT TO THE PROPAGATION PATHS FOR A PHASE-TO-GROUND SOLID FAULT LOCATED AT Bus 812 of Fig. 1. The Analysis Refers to the Propagation Mode 0

\begin{tabular}{|c|c|c|c|c|}
\hline \multirow{2}{*}{ Path } & \multirow{2}{*}{$\begin{array}{l}\text { Path } \\
\text { length } \\
n_{p} \cdot L_{p} \\
(\mathrm{~km})\end{array}$} & \multirow{2}{*}{$\begin{array}{l}\text { Theoretical } \\
\text { frequencies } f_{p, i} \\
\text { (traveling wave refer } \\
\text { to propagation } \\
\text { mode 0) } \\
(\mathrm{kHz})\end{array}$} & \multicolumn{2}{|c|}{$\begin{array}{l}\text { CWT identified frequency } \\
\qquad(\mathrm{kHz})\end{array}$} \\
\hline & & & $\begin{array}{c}\text { Transient- } \\
\text { based mother } \\
\text { wavelet }\end{array}$ & $\begin{array}{l}\text { Morlet } \\
\text { mother } \\
\text { wavelet }\end{array}$ \\
\hline $800-812$ & $4 \times 22.57$ & 2.98 & 3.90 & 3.80 \\
\hline $800-808$ & $4 \times 11.14$ & 6.04 & 6.00 & - \\
\hline $800-810$ & $2 \times 12.92$ & 10.42 & 13.00 & 9.40 \\
\hline
\end{tabular}

kHz [see Fig. 8(b)]. The parameters adopted for the $50 \mathrm{~Hz}$ part of the transformer model are the following: 5 MVA $150 / 24.9 \mathrm{kV}$ $V_{\mathrm{sc}}=9 \%$ for the substation; $1 \mathrm{MVA} 24.9 / 0.4 \mathrm{kV} V_{\mathrm{sc}}=4 \%$ for the loads and 2.5 MVA $24.9 / 24.9 \mathrm{kV} V_{\mathrm{sc}}=8 \%$ for the loads.

A constant parameter line model (CP-line model) is adopted for the representation of the overhead lines [22]. The adoption of a frequency dependent line model (e.g., the FD-line model [22]) results in voltage transients close to those obtained with the CP-line model. This is due on the one hand to the typically limited length of distribution lines and, on the other hand, to the typical frequency content of the fault transients, which does not exceed a few tens of $\mathrm{kHz}$.

Table III shows the modal parameters of the CP-line model that refers to the considered overhead line configuration. The modal parameters are calculated for a frequency equal to $1 \mathrm{kHz}$ and assuming the ground resistivity equal to $100 \Omega \mathrm{m}$.

\section{B. Unbalanced Grounded Faults}

The type of fault (balanced or unbalanced) influences the CWT signal energy $E_{\text {cwt }}(a)$ relevant to each propagation mode. In particular, for the case of unbalanced faults, the propagation mode 0 always results, as expected, in the larger energy content. Therefore, the propagation mode 0 (ground mode) is the CWT-analyzed one for the case of unbalanced grounded faults.

Let consider a phase-to-ground solid fault located in correspondence of bus 812 of Fig. 1. For this fault location, three characteristic frequencies can be identified. Table IV shows the comparison between the results obtained with the CWT analysis using the fault-inferred and Morlet mother wavelets. Also for the case of unbalanced faults, the proposed approach, suitably combined with the use of transient-based mother wavelet, allows for the identification of all the characteristic frequencies.

\section{Effect of the Fault Impedance}

In this section, the effect of the fault impedance on the accuracy of the fault location algorithm is analyzed.

A phase-to-ground fault has been assumed at bus 806 of the IEEE 34-bus distribution system. Such a bus has been selected in order to analyze a fault location characterized by a single path. Three different fault impedances have been considered, namely 0,10 and $100 \Omega$ and the results are shown in Table V. Such a table also reports the location accuracy, $\Delta d$, calculated as the difference between the length of the path $p *$, between the 
TABLE V

CharacteristiC AND CWT IDENTIFIED FREQUENCIES RELEVANT to THE PROPAGATION PATHS FOR A PHASE-TO-GROUND FAULT LOCATED AT BUS 806 of Fig. 1. The Results MaKe REFERENCE TO THE TRANSIENT-BASED Mother WaVelet. The Analysis Refers to the Propagation Mode 0

\begin{tabular}{|c|c|c|c|}
\hline $\begin{array}{c}\text { Fault } \\
\text { Impedance } \\
(\Omega)\end{array}$ & $\begin{array}{c}\text { Theoretical frequencies } f_{p, i} \\
\text { (traveling wave refer to } \\
\text { propagation mode 0) } \\
(\mathrm{kHz})\end{array}$ & $\begin{array}{c}\text { CWT identified } \\
\text { frequency } \\
(\mathrm{kHz})\end{array}$ & $\begin{array}{c}\Delta d \\
(\mathrm{~m})\end{array}$ \\
\hline 0 & 51.00 & 50.20 & 21.0 \\
\hline 10 & 51.00 & 50.20 & 21.0 \\
\hline 100 & 51.00 & 51.60 & 15.3 \\
\hline
\end{tabular}

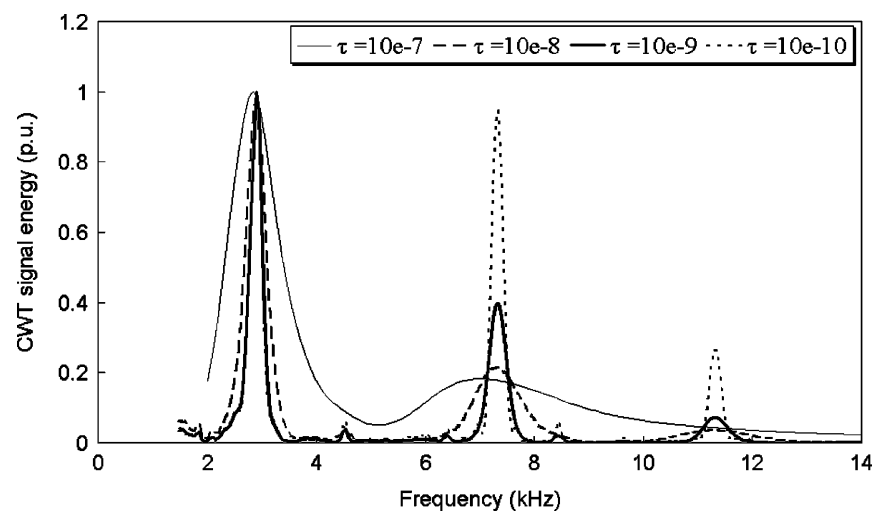

Fig. 9. Comparison between the results of the CWT analysis performed with different mother wavelet exponential decay time. Analysis performed in the mode 1 of the voltage transient recorded at bus 800 for a three-phase zeroimpedance fault at bus 814 . The values are in per-unit with respect to the maximum.

observation bus and the fault location, and the CWT-identified length of the same path.

As it can be seen, the accuracy of the proposed algorithm is not influenced by the different fault impedance values. This result can be explained by considering that the variation of the fault impedance results in a variation of the value of the reflection coefficient corresponding to the fault location but not in its sign. Indeed, this last is the only responsible for a change in the characteristic frequency of the fault path.

\section{Influence of the Mother Wavelet Exponential Decay Time}

In this section, the influence of the exponential decay time $\tau$ of the mother wavelet expressed by (8) is analyzed. In particular, four different values of $\tau$ have been considered, namely: $10^{-7}, 10^{-8}, 10^{-9}$ and $10^{-10}$. The relevant CWT analysis of the voltage transient recorded at bus 800 for a three-phase solid fault at bus 814 is reported in Fig. 9 .

The results show that the increase of the exponential decay time of the mother wavelet results in a decrease of the algorithm performances in the identification of characteristic frequencies of larger amplitudes. This result can be justified by considering that the lowest is the exponential time decay, the larger is the extension of the frequency spectrum of the daughter wavelet that result in an increase of the matching between the frequencies contained in the analyzed signal with the ones of the daughter wavelets.
TABLE VI

Characteristic AND CWT IDENTIFIED FREQUENCIES OF THREe-PHASE SOLID FAULTS IN SOME BUSES OF THE IEEE 34-Bus DistRIBUTION SySTEM REPORTED IN Fig. 8(A). THE RESUlts MAKE REFERENCE TO THE TRANSIENT-INFERRED MOTHER WAVELET

\begin{tabular}{|c|c|c|c|}
\hline $\begin{array}{c}\text { Fault location } \\
\text { bus }\end{array}$ & $\begin{array}{c}\text { Theoretical freq } \\
(\mathrm{kHz})\end{array}$ & $\begin{array}{c}\text { CWT identified } \\
\text { frequency } \\
(\mathrm{kHz})\end{array}$ & $\begin{array}{c}\Delta d \\
(\mathrm{~m})\end{array}$ \\
\hline 806 & 56.76 & 54.40 & 57.30 \\
\hline 808 & 6.73 & 7.45 & -1083 \\
\hline 810 & 5.8 & 6.55 & -1481 \\
\hline 812 & 3.32 & 3.80 & -2853 \\
\hline 834 & 22.75 & 21.20 & 239.7 \\
\hline 836 & 15.80 & 13.70 & 721 \\
\hline 838 & 11.85 & 10.40 & 874.3 \\
\hline 840 & 15.08 & 13.20 & 706.1 \\
\hline 842 & 22.14 & 20.70 & 234.4 \\
\hline 844 & 19.87 & 17.30 & 560.9 \\
\hline 846 & 15.35 & 12.3 & 1211.5 \\
\hline 848 & 14.76 & 11.60 & 1374.2 \\
\hline 858 & 49.94 & 48.60 & 40.96 \\
\hline 860 & 19.26 & 17.60 & 367.1 \\
\hline 862 & 15.61 & 13.40 & 791.4 \\
\hline 864 & 37.84 & 37.90 & -3.09 \\
\hline
\end{tabular}

TABLE VII

CHARACTERISTIC AND CWT IDENTIFIED FREQUENCIES OF PHASE-TO-GROUND FAUlTS IN SOME Buses OF THE IEEE 34-Bus DistRIBUTION SYSTEM REPORTED IN Fig. 8(A). THE RESUlts MAKe REFERENCE TO THE TRANSIENT-INFERRED MOTHER WAVELET

\begin{tabular}{|c|c|c|c|}
\hline $\begin{array}{c}\text { Fault location } \\
\text { bus }\end{array}$ & $\begin{array}{c}\text { Theoretical freq } \\
(\mathrm{kHz})\end{array}$ & $\begin{array}{c}\text { CWT identified } \\
\text { frequency } \\
(\mathrm{kHz})\end{array}$ & $\begin{array}{c}\Delta d \\
(\mathrm{~m})\end{array}$ \\
\hline 806 & 51.00 & 50.20 & 21 \\
\hline 808 & 6.68 & 7.50 & -1219.3 \\
\hline 810 & 5.76 & 6.60 & -1646.5 \\
\hline 812 & 3.32 & 3.80 & -2853 \\
\hline 834 & 20.59 & 19.30 & 218.2 \\
\hline 836 & 14.29 & 13.00 & 468.7 \\
\hline 838 & 10.72 & 9.70 & 660.5 \\
\hline 840 & 13.55 & 12.20 & 548.2 \\
\hline 842 & 20.04 & 19.00 & 183.3 \\
\hline 844 & 17.86 & 15.80 & 490.9 \\
\hline 846 & 13.80 & 11.30 & 1077.7 \\
\hline 848 & 13.36 & 11.00 & 1080.2 \\
\hline 858 & 45.18 & 44.90 & 9.39 \\
\hline 860 & 17.31 & 16.20 & 265.7 \\
\hline 862 & 14.03 & 12.70 & 500.9 \\
\hline 864 & 34.00 & 35.60 & -88.9 \\
\hline
\end{tabular}

\section{E. Effect of Variation of Fault Position Along the IEEE 34-Bus Distribution System}

In what follows we extend our analysis by applying the proposed algorithm to the location of single/three phase faults at all the buses of the IEEE 34-bus distribution system. The results relevant to the three-phase fault are reported in Table VI while the results of single phase faults in Table VII. Both tables also report the location accuracy, $\Delta d$, calculated following the same procedure described in previous Section IV.

It can be observed that the proposed algorithm performs satisfactorily for any of the fault positions. 


\section{CONCLUSION}

The paper has presented an improved version of a fault location algorithm developed at the authors laboratory [13] based on the analysis of fault-generated traveling waves by means of the CWT. The improvement that has been here presented, discussed and tested, is based on the appropriate definition and use of mother wavelets inferred from fault transient waveforms. The definition of these mother wavelets allows indeed one to overcome some limitation of the original algorithm, which was related to the use of traditional mother wavelets (e.g., the Morlet one). In particular, the use of traditional mother wavelets does not allow, in general, to identify all the characteristic frequencies of the fault-originated traveling waves, along with the relevant paths, associated with a specific fault location. This has been confirmed by means of an extensive analysis carried out on the IEEE 34-bus distribution system.

A comprehensive evaluation of the performance of the proposed algorithm has been presented for different types of fault, of their impedance and positions by making use of an advanced simulation tool. The overall effectiveness of the proposed algorithm has been proved.

Future research efforts will be devoted, on the one hand, to the orthogonalization process of transient-inferred mother wavelet in order to allow for the reconstruction of the transient signal for each characteristic frequency: this is expected to improve the algorithm accuracy by means of proper integration of time-domain fault location approaches [29]. On the other hand, the authors feel worth adding that this paper represents the theoretical basis for a fault location tool/device, which is expected to be the object of a future research project.

\section{ACKNOWLEDGMENT}

The authors would like to thank Prof. A. Abur for useful discussions on the subject.

This paper takes into account the comments received during the presentation of a preliminary version at the 7th International Conference on Power System Transients (IPST'07), Lyon, France, June 4-7, 2007, and of the reviewers.

\section{REFERENCES}

[1] Fault Management in Electrical Distribution Systems, CIRED WG03 Fault Management, 1998.

[2] M. S. Sachdev and R. Agarwal, "A technique for estimating transmission line fault locations from digital impedance relay measurements," IEEE Trans. Power Del., vol. 3, no. 1, pp. 121-129, Jan. 1988.

[3] K. Srinivasan and A. St.-Jacques, "A new fault location algorithm for radial transmission lines with loads," IEEE Trans. Power Del., vol. 4, no. 3, pp. 1676-1682, Jul. 1989.

[4] A. A. Girgis, D. G. Hart, and W. L. Peterson, "A new fault location technique for two- and three-terminal lines," IEEE Trans. Power Del., vol. 7, no. 1, pp. 98-107, Jan. 1992.

[5] G. B. Ancell and N. C. Pahalawatha, "Maximum likelihood estimation of fault location on transmission lines using travelling waves," IEEE Trans. Power Del., vol. 9, no. 2, pp. 680-689, Apr. 1994.

[6] O. Chaari, M. Meunier, and F. Brouaye, "Wavelets: A new tool for the resonant grounded power distribution systems relaying," IEEE Trans. Power Del., vol. 11, no. 3, pp. 1301-1308, Jul. 1996.

[7] F. H. Magnago and A. Abur, "Fault location using wavelets," IEEE Trans. Power Del., vol. 13, no. 4, pp. 1475-1480, Oct. 1998.

[8] F. H. Magnago and A. Abur, "A new fault location technique for radial distribution systems based on high frequency signals," in Proc. IEEE-Power Eng. Soc. Summer Meeting, Jul. 18-22, 1999, vol. 1, pp. $426-431$.
[9] D. W. P. Thomas, R. E. Batty, C. Christopoulos, and A. Wang, "A novel transmission-line voltage measuring method," IEEE Trans. Instrum. Meas., vol. 47, no. 5, pp. 1265-1270, Oct. 1998.

[10] S. Ebron, D. L. Lubkeman, and M. White, "A neural network approach to the detection of incipient faults on power distribution feeders," IEEE Trans. Power Del., vol. 5, no. 2, pp. 905-914, Apr. 1990.

[11] Z. Chen and J. C. Maun, "Artificial neural network approach to singleended fault locator for transmission lines," IEEE Trans. Power Syst., vol. 15, no. 1, pp. 370-375, Feb. 2000.

[12] N. Kandil, V. K. Sood, K. Khorasani, and R. V. Patel, "Fault identification in an AC-DC transmission system using neural networks," IEEE Trans. Power Syst., vol. 7, no. 2, pp. 812-819, May 2002.

[13] A. Borghetti, S. Corsi, C. A. Nucci, M. Paolone, L. Peretto, and R. Tinarelli, "On the use of continuous-wavelet transform for fault location in distribution power networks," Elect. Power Energy Syst., vol. 28, pp. 608-617, 2006.

[14] P. Goupillaud, A. Grossmann, and J. Morlet, "Cycle-octave and related transforms in seismic signal analysis," Geoexploration, vol. 23, pp. 85-102, 1984-1985.

[15] I. Daubechies, "The wavelet transform, time-frequency localization and signal analysis," IEEE Trans. Inf. Theory, vol. 36, no. 9, pp. 961-1005, Sep. 1990.

[16] I. Daubechies, "Orthonormal bases of compactly supported wavelets," Commun. Pure Appl. Math., vol. XLI, pp. 909-996, 1988.

[17] S. G. Mallat, "A theory for multiresolution signal decomposition: The wavelet representation," IEEE Trans. Pattern Anal. Mach. Intell., vol. 11, no. 7, pp. 674-693, Jul. 1989.

[18] S. G. Mallat, "Multifrequency channel decompositions of images and wavelet models," IEEE Trans. Acoust., Speech, Signal Process., vol. ASSP-37, no. 12, pp. 2091-2110, Dec. 1989.

[19] O. Rioul and M. Vetterli, "Wavelets and signal processing," IEEE Signal Process. Mag., vol. 8, no. 4, pp. 14-38, Oct. 1991.

[20] IEEE Distribution Planning Working Group, "Radial distribution test feeders," IEEE Trans. Power Syst., vol. 6, no. 3, pp. 975-985, Aug. 1991.

[21] E. Clarke, Circuit Analysis of AC Power Systems, 1. New York: Wiley, 1943.

[22] H. W. Dommel, "Digital computer solution of electromagnetic transients in single and multi-phase networks," IEEE Trans. Power App. Syst., vol. PAS-88, pp. 388-399, Apr. 1969

[23] A. V. Oppenheim and R. W. Schafer, Discrete-Time Signal Processing. Englewood Cliffs, NJ: Prentice-Hall, 1989.

[24] A. Graps, "An introduction to wavelets," IEEE Comput. Sci. Eng., vol. 2, no. 2, pp. 50-61, Summer, 1995.

[25] T. Lobos, T. Sikorski, and P. Schegner, "Joint time-frequency representation of non-stationary signals in electrical power engineering," in Proc. 15th Power Systems Computation Conf. (PSCC'05), Liege, Belgium, Aug. 22-26, 2005, paper fp 97.

[26] J. Mahseredjian, L. Dubé, and L. Gérin-Lajoie, "New advances in the simulation of transients with EMTP: Computation and visualization techniques," in Proc. 7th Int. Conf. Modeling and Simulation of Electric Machines, Converters and Systems, Montreal, QC, Canada, Aug. 2002.

[27] J. Mahseredjian, S. Dennetière, L. Dubé, and B. Khodabakhchian, "On a new approach for the simulation of transients in power systems," in Proc. Int. Conf. Power Systems Transients IPST'2005, Montreal, QC, Canada, Jun. 2005.

[28] G. Strang and T. Nguyen, Wavelets and Filter Banks. Wellesley, MA Wellesley Cambridge Press, 1996

[29] A. Abur, Private Communication.

Alberto Borghetti (M'97-SM'03) was born in Cesena, Italy, in 1967. He graduated (with honors) in electrical engineering from the University of Bologna, Bologna, Italy, in 1992.

Since then, he has been working with the Power System Group at the University of Bologna, where he was appointed Researcher in 1994 and Associate Professor of electric power systems in 2004. His main research interests concern power system analysis, with particular reference to voltage collapse, power system restoration, electromagnetic transients, and optimal generation scheduling.

Mr. Borghetti is a member of the IEEE PES Working Group on Distributed Resources: Modeling and Analysis, of the IEEE Working Group on Lightning Performance of Distribution Lines, of the joint CIGRE-CIRED Working Group "Protection of MV and LV networks against Lightning," and of the CIGRE Working Group C4.6.01 on Power System Security Assessment. He is the Italian representative to the European COST Action P18 "The Physics of Lightning Flash and its Effects." 
Mauro Bosetti was born in Trento, Italy, in 1978. He received the degree with honors in electrical engineering in 2005 at the University of Bologna, Bologna, Italy, where he is currently pursuing the Ph.D. degree within the power system group.

His research interests are distributed generation, with particular reference to micro-grid operation, and fault location.

Mauro Di Silvestro was born in Giulianova, Italy, in 1980. He received the degree with honors in electrical engineering at the University of Bologna, Bologna, Italy, in 2005.

He is currently working within the Power Systems Group of the University of Bologna. His research interests are power system transients, with particular reference to fault location and distributed generation.

Carlo Alberto Nucci (M'91-SM'02-F'07) was born in Bologna, Italy, in 1956. $\mathrm{He}$ received the M.S. (hons.) and Ph.D. degrees in electrical engineering from the University of Bologna in 1982 and 1986, respectively.

At the University of Bologna, he was researcher at the Power Electrical Engineering Institute since 1983, became an Associate Professor in 1992, and in
2000 became a full Professor and Chair of power systems. He is author or coauthor of more than 200 scientific papers published on reviewed journals or presented at international conferences. His research interests concern power systems transients and dynamics, with particular reference to lightning impact on power lines, system restoration after black-out, and distributed generation.

Dr. Nucci is member of the IEEE Working Group on Lightning Performance of Distribution Lines. In CIGRE, he serves as chairman of the Study Committee C4 "System Technical performance." He is also Fellow of the IET.

Mario Paolone (M'07) was born in Campobasso, Italy, in 1973. He received the degree with honors in electrical engineering in 1998 and the Ph.D. degree in 2002 from the University of Bologna, Bologna, Italy.

$\mathrm{He}$ is currently working within the Power Systems Group at the University of Bologna. His research interests are power system transients, with particular reference to LEMP-interaction with electrical networks, power systems dynamics, power system protections, and distributed generation.

Dr. Paolone is member of the IEEE Working Group on Lightning Performance of Distribution Lines and of the joint CIGRE-CIRED Working Group on Protection of MV and LV Networks Against Lightning." 